\title{
A Viscous Fluid Model for Multimodal Non-rigid Image Registration Using Mutual Information
}

\author{
E. D’Agostino, F. Maes`, D. Vandermeulen, and P. Suetens \\ Katholieke Universiteit Leuven \\ Faculties of Medicine and Engineering \\ Medical Image Computing (Radiology - ESAT/PSI) \\ University Hospital Gasthuisberg, Herestraat 49, B-3000 Leuven, Belgium \\ Emiliano.DAgostino@uz.kuleuven.ac.be
}

\begin{abstract}
We propose a multimodal free form registration algorithm based on maximization of mutual information. Images to be aligned are modeled as a viscous fluid that deforms under the influence of forces derived from the gradient of the mutual information registration criterion. Parzen windowing is used to estimate the joint intensity probability of the images to be matched. The method was verified by for registration of simulated T1-T1, T1-T2 and T1-PD images with known ground truth deformation. The results show that the root mean square difference being the recovered and the ground truth deformation is smaller than 1 voxel.
\end{abstract}

\section{Introduction}

Maximization of mutual information has been demonstrated to be a very general and reliable approach for affine registration of multimodal images of the same patient or from different patients, including atlas matching [7,9]. In applications where local morphological differences need to be quantified, affine registration is no longer sufficient and non-rigid registration (NRR) is required, aiming at finding a $3 \mathrm{D}$ vector field describing the deformation at each point. Applications for NRR include shape analysis (to warp all shapes to a standard space) and atlas-based segmentation (to compensate for gross morphological differences between atlas and study images). Different approaches have been proposed for extending the mutual information criterion to NRR. Spline-based approaches [8] can correct for gross shape differences, but a dense grid of control points is required to characterize the deformation at voxel level detail, implying high computational complexity. Block matching [4] or free-form approaches, using a non-parameterized expression for the deformation field, assign a local deformation vector to each voxel individually, but need appropriate constraints for spatial regularization of the resulting vector field. Elastic constraints are suitable when displacements can be assumed to be small, while for large magnitude deformations a viscous fluid model is more appropriate.

\footnotetext{
* Frederik Maes is Postdoctoral Fellow of the Fund for Scientific Research - Flanders (FWO-Vlaanderen, Belgium).
} 
Recently, a multimodal NRR algorithm was presented in [5], defining the forces driving the deformation at each voxel such that mutual information is maximized and using a regularization functional derived from linear elasticity theory. In this paper, we extend the approach of $[5]$ by replacing the elastic model by the viscous fluid regularization model of Christensen et al. 3] and thus generalize the method of [3] to multimodal image registration based on maximization of mutual information. The Navier-Stokes equation modelling the viscous fluid is solved by iteratively updating the deformation field and convolving it with a Gaussian filter. The deformation field is regridded as needed during iterations as in 3 to assure that its Jacobian remains positive everywhere, such that the method can handle large deformations. We verified the robustness of the method by applying realistic known deformations to simulated multispectral MR images and evaluating the difference between the recovered and ground truth deformation fields in terms of displacement errors and of tissue classification errors when using the recovered deformation for atlas-based segmentation.

\section{Method}

\subsection{The Viscous Fluid Algorithm}

We follow the approach of [3] to deform an template image $\mathcal{F}$ onto a target image $\mathcal{G}$, using an Eulerian reference frame to represent the mapping $\boldsymbol{T}=\boldsymbol{x}-\boldsymbol{u}(\boldsymbol{x})$ of fixed voxel positions $\boldsymbol{x}$ in target space onto the corresponding positions $\boldsymbol{x}-$ $\boldsymbol{u}(\boldsymbol{x})$ in the original template space. The deforming template image is considered as a viscous fluid whose motion is governed by the Navier-Stokes equation of conservation of momentum. Using the same simplifications as in [3], this equation can be written as

$$
\nabla^{2} \boldsymbol{v}+\nabla(\nabla . \boldsymbol{v})+\boldsymbol{F}(\boldsymbol{x}, \boldsymbol{u})=0
$$

with $\boldsymbol{F}(\boldsymbol{x}, \boldsymbol{u})$ a force field acting at each position $\boldsymbol{x}$ that depends on the deformation $\boldsymbol{u}$ and that drives the deformation in the appropriate direction, and with $\boldsymbol{v}(\boldsymbol{x}, t)$ the deformation velocity experienced by a particle at position $\boldsymbol{x}$ :

$$
\boldsymbol{v}=\frac{d \boldsymbol{u}}{d t}=\frac{\partial \boldsymbol{u}}{\partial t}+\sum_{i=1}^{3} v_{i} \frac{\partial \boldsymbol{u}}{\partial x_{i}}
$$

with $\boldsymbol{v}=\left[v_{1}(\boldsymbol{x}, t), v_{2}(\boldsymbol{x}, t), v_{3}(\boldsymbol{x}, t)\right]^{T}$ and $\boldsymbol{u}=\left[u_{1}(\boldsymbol{x}, t), u_{2}(\boldsymbol{x}, t), u_{3}(\boldsymbol{x}, t)\right]^{T}$.

In section 2.2 , we derive an expression for the force field $\boldsymbol{F}$ such that the viscous fluid flow maximizes mutual information between corresponding voxel intensities. When the forces are given, solving (11) yields deformation velocities, from which the deformation itself can be computed by integration over time. In [3] the Navier-Stokes equation is solved by Successive Over Relaxation (SOR), but this is a computationally expensive approach. Instead, we follow the approach of [2] and obtain the velocity field by convolution of the force field with a Gaussian kernel $\psi$ :

$$
\boldsymbol{v}=\psi \star \boldsymbol{F}
$$


The displacement $\boldsymbol{u}^{(k+1)}$ at iteration $(k+1)$ is then given by:

$$
\boldsymbol{u}^{(k+1)}=\boldsymbol{u}^{(k)}+\boldsymbol{R}^{(k)} \cdot \Delta t
$$

with $\boldsymbol{R}^{(k)}$ the perturbation to the deformation field:

$$
\boldsymbol{R}^{(k)}=\boldsymbol{v}^{(k)}-\sum_{i=1}^{3} v_{i}^{(k)}\left[\frac{\partial \boldsymbol{u}^{(k)}}{\partial x_{i}}\right]
$$

The time step $\Delta t$ is constrained by $\Delta t \leq \max (\|\boldsymbol{R}\|) \cdot \Delta u$, with $\Delta u$ the maximal voxel displacement that is allowed in one iteration.

To preserve the topology of the object image, the Jacobian of the deformation field should not become negative. When the Jacobian becomes anywhere smaller than some positive threshold, regridding of the deformed template image is applied as in 3. to generate a new template, setting the incremental displacement field to zero. The total deformation is the concatenation of the incremental deformation fields associated with each propagated template.

\section{$2.2 \quad$ Force Field Definition}

We define an expression for the force field $\boldsymbol{F}(\boldsymbol{x}, \boldsymbol{u})$ in (1) such that the viscous fluid deformation strives at maximizing mutual information $I(\boldsymbol{u})$ of corresponding voxel intensities between the deformed template image $\mathcal{F}(\boldsymbol{x}-\boldsymbol{u})$ and the target image $\mathcal{G}(\boldsymbol{x})$. We adopt here the approach of [5] who derived an expression for the gradient $\nabla_{\boldsymbol{u}} I$ of $I$ with respect to the deformation field $\boldsymbol{u}$, modelling the joint intensity distribution $\left.p_{\boldsymbol{u}}^{\mathcal{F}} \mathcal{G}_{\left(i_{1}\right.}, i_{2}\right)$ of template and target images as a continuous function using Parzen windowing. If the deformation field $\boldsymbol{u}$ is perturbed into $\boldsymbol{u}+\epsilon \boldsymbol{h}$, variational calculus yields the first variation of $I$ :

$$
\begin{aligned}
\left.\frac{\partial I(\boldsymbol{u}+\epsilon \boldsymbol{h})}{\partial \epsilon}\right|_{\epsilon=0} & =\iint \frac{\partial}{\partial \epsilon}\left[p_{\boldsymbol{u}+\epsilon \boldsymbol{h}}^{\mathcal{F}, \mathcal{G}}\left(i_{1}, i_{2}\right) \log \frac{p_{\boldsymbol{u}+\epsilon \boldsymbol{h}}^{\mathcal{F}, \mathcal{G}}\left(i_{1}, i_{2}\right)}{p^{\mathcal{F}}\left(i_{1}\right) p_{\boldsymbol{u}+\epsilon \boldsymbol{h}}^{\mathcal{G}}\left(i_{2}\right)}\right]_{\epsilon=0} d i_{1} d i_{2} \\
& =\left.\iint\left(1+\log \frac{p_{\boldsymbol{u}}^{\mathcal{F}, \mathcal{G}}\left(i_{1}, i_{2}\right)}{p^{\mathcal{F}}\left(i_{1}\right) p_{\boldsymbol{u}} \mathcal{G}\left(i_{2}\right)}\right) \frac{\partial p_{\boldsymbol{u}+\epsilon \boldsymbol{h}}^{\mathcal{F}, \mathcal{G}}\left(i_{1}, i_{2}\right)}{\partial \epsilon}\right|_{\epsilon=0} d i_{1} d i_{2}(6)
\end{aligned}
$$

The joint intensity probability is constructed from the domain of overlap $\mathcal{V}$ of both images (with volume $V$ ), using the Parzen windowing kernel $\psi\left(i_{1}, i_{2}\right)$ :

$$
p_{\boldsymbol{u}}^{\mathcal{F}, \mathcal{G}}\left(i_{1}, i_{2}\right)=\frac{1}{V} \int_{\mathcal{V}} \psi\left(i_{1}-\mathcal{F}(\boldsymbol{x}-\boldsymbol{u}), i_{2}-\mathcal{G}(\boldsymbol{x})\right) d \boldsymbol{x}
$$

Inserting (77) in (6) and rearranging as in [5], yields

$$
\left.\frac{\partial I(\boldsymbol{u}+\epsilon \boldsymbol{h})}{\partial \epsilon}\right|_{\epsilon=0}=\frac{1}{V} \int_{\mathcal{V}}\left[\psi \star \frac{\partial L_{\boldsymbol{u}}}{\partial i_{1}}\right](\mathcal{F}(\boldsymbol{x}-\boldsymbol{u}), \mathcal{G}(\boldsymbol{x})) \nabla \mathcal{F}(\boldsymbol{x}-\boldsymbol{u}) \boldsymbol{h}(\boldsymbol{x}) d \boldsymbol{x}
$$

with

$$
L_{\boldsymbol{u}}\left(i_{1}, i_{2}\right)=1+\log \frac{p_{\boldsymbol{u}}^{\mathcal{F}, \mathcal{G}}\left(i_{1}, i_{2}\right)}{p^{\mathcal{F}}\left(i_{1}\right) p_{\boldsymbol{u}}^{\mathcal{G}}\left(i_{2}\right)}
$$



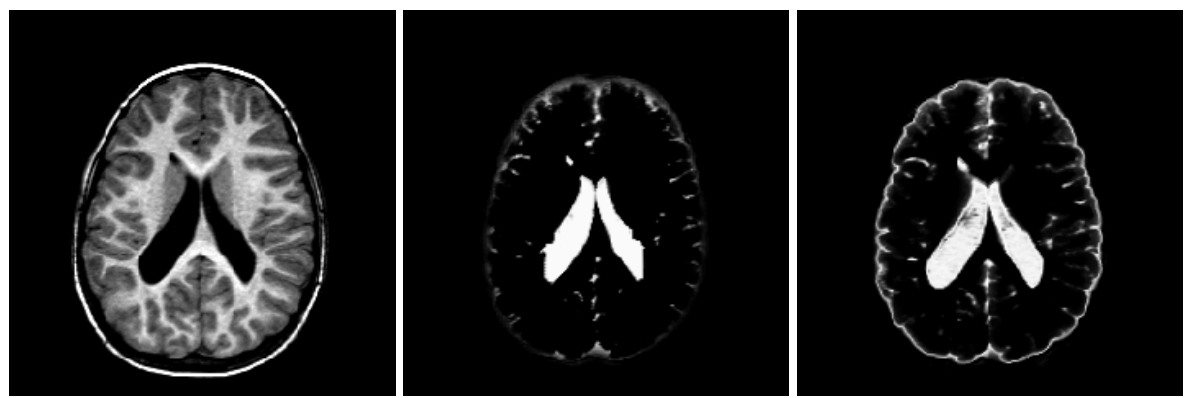

Fig. 1. Left: T1 MPRAGE Patient image; Middle: CSF segmented using standard prior; Right: CSF segmented after non-rigid matching of the atlas.

Table 1. Root mean square error $\Delta T$ in millimeter between ground thruth and recovered deformation fields within the brain region for different multimodal image combinations of BrainWeb simulated MR brain images at different noise levels.

\begin{tabular}{|c|ccc|ccc|c|}
\hline Case & \multicolumn{3}{|c|}{$\mathrm{T} 1 / \mathrm{T} 1$} & \multicolumn{3}{c|}{$\mathrm{T} 1 / \mathrm{T} 2$} & $\mathrm{~T} 1 / \mathrm{PD}$ \\
\hline & $0 \%$ & $3 \%$ & $7 \%$ & $0 \%$ & $3 \%$ & $7 \%$ & $3 \%$ \\
\hline 1 & 0.384 & 0.430 & 0.465 & 0.577 & 0.759 & 0.685 & 0.723 \\
\hline 2 & 0.304 & 0.398 & 0.433 & 0.443 & 0.640 & 0.649 & 0.661 \\
\hline 3 & 0.351 & 0.411 & 0.459 & 0.505 & 0.753 & 0.775 & 0.772 \\
\hline
\end{tabular}

We therefore define the force field $\boldsymbol{F}$ at $\boldsymbol{x}$ to be equal to the gradient of $I$ with respect to $\boldsymbol{u}(\boldsymbol{x})$, such that $\boldsymbol{F}$ drives the deformation to maximize $I$ :

$$
\boldsymbol{F}(\boldsymbol{x}, \boldsymbol{u})=\nabla_{\boldsymbol{u}} I=\frac{1}{V}\left[\psi \star \frac{\partial L^{\boldsymbol{u}}}{\partial i_{1}}\right](\mathcal{F}(\boldsymbol{x}-\boldsymbol{u}), \mathcal{G}(\boldsymbol{x})) \nabla \mathcal{F}(\boldsymbol{x}-\boldsymbol{u})
$$

\subsection{Implementation Issues}

The method was implemented in Matlab, with the image resampling and histogram computation coded in C. The histogram was computed using 128 bins for both template and target images. Parzen windowing was performed by convolution of the joint histogram with a 2D Gaussian kernel. The maximal displacement at each iteration $\Delta u$ was set to 0.3 voxels and regridding was performed when the Jacobian became smaller than 0.5. Iterations were continued as long as mutual information $I(\boldsymbol{u})$ increased, with a maximum of 75 iterations. A multiresolution optimization strategy was adopted by smoothing and downsampling the images at 3 different levels of resolution, starting the process at the coarsest level and gradually increassing resolution as the method converged. Computation time for matching two images of size $128 \times 128 \times 80$ is about 50 minutes. 
Table 2. Overlap coefficient for different tissue classes of tissue maps obtained with ground thruth and recovered deformation fields for different multimodal image combinations of BrainWeb simulated MR brain images. Noise level was $3 \%$ in each case.

\begin{tabular}{|c|ccc|ccc|ccc|}
\hline Case & \multicolumn{3}{|c|}{ T1/T1 } & \multicolumn{3}{c|}{ T1/T2 } & \multicolumn{3}{c|}{ T1/PD } \\
\hline & WM & GM & CSF & WM & GM & CSF & WM & GM & CSF \\
\hline 1 & 0.9282 & 0.9179 & 0.8698 & 0.8579 & 0.8320 & 0.7645 & 0.8604 & 0.8454 & 0.7844 \\
\hline 2 & 0.9253 & 0.9277 & 0.8969 & 0.8595 & 0.8463 & 0.7839 & 0.8564 & 0.8373 & 0.7818 \\
\hline 3 & 0.9279 & 0.9260 & 0.8795 & 0.8460 & 0.8270 & 0.7579 & 0.8552 & 0.8028 & 0.7413 \\
\hline
\end{tabular}

\section{Experiments}

The method was validated on simulated images generated by the BrainWeb MR simulator [1] with different noise levels. In all experiments the images were non-rigidly deformed by known deformation fields $\boldsymbol{T}^{*}$. These were generated by using our method to match the T1 weighted BrainWeb image to real T1 weighted images of 3 periventricular leukomalacia patients, typically showing enlarged ventricles. We evaluate how well the recovered deformation $\boldsymbol{T}$, obtained by matching the original $\mathrm{T} 1$ weighted BrainWeb image to the $\mathrm{T} 1, \mathrm{~T} 2$ or proton density (PD) weighted images deformed by $\boldsymbol{T}^{*}$, resembles the ground truth $\boldsymbol{T}^{*}$. Both deformations were compared by their root mean square (RMS) error $\Delta T$ evaluated in millimeter over all brain voxels $\mathcal{B}$ :

$$
\Delta T=\sqrt{\frac{1}{N_{\mathcal{B}}} \sum_{\mathcal{B}}\left(\left|\boldsymbol{T}(\boldsymbol{x})-\boldsymbol{T}^{*}(\boldsymbol{x})\right|\right)^{2}}
$$

We also verified the impact of possible registration errors on atlas-based segmentation by comparing the (hard classified) tissue maps $M$ and $M^{*}$, obtained by deforming the tissue maps of the original image using $\boldsymbol{T}$ and $\boldsymbol{T}^{*}$ respectively. We measure the difference between $M$ and $M^{*}$ by their overlap coefficient $O_{j}\left(M, M^{*}\right)$ for 3 tissue types $j$, white matter (WM), grey matter (GM) and cerebro-spinal fluid (CSF):

$$
O_{j}\left(M, M^{*}\right)=\frac{2 V_{j}\left(M, M^{*}\right)}{V_{j}(M)+V_{j}\left(M^{*}\right)}
$$

with $V_{j}\left(M, M^{*}\right)$ the volume of the voxels that are assigned to class $j$ in both maps and $V_{j}(M)$ and $V_{j}\left(M^{*}\right)$ the volume of the voxels assigned to class $j$ in each map separately.

Figure 1 shows the registration result of the BrainWeb T1 image to one of the patient images and the segmentation of CSF obtained using the method of [9] with affine and with our non-rigid atlas registration procedure. Note how the segmentation of the enlarged ventricles is much improved by using non-rigid atlas warping.

Table 1 shows the RMS error $\Delta T$ computed for $\mathrm{T} 1$ to $\mathrm{T} 1$, T2 and PD registration of the BrainWeb images at different noise levels (each time identical for 

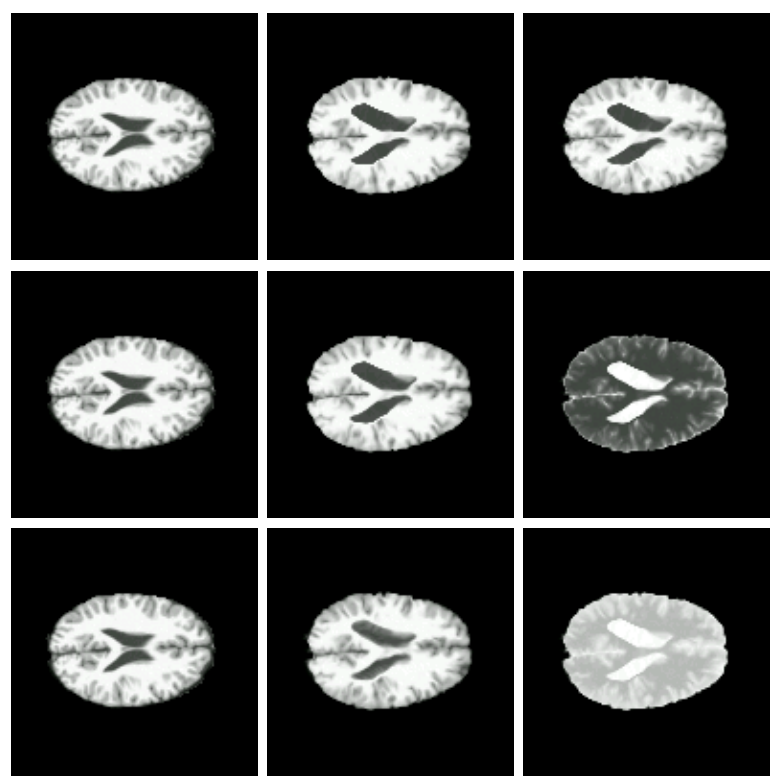

Fig. 2. Left: Original BrainWeb T1 template; right: BrainWeb target image obtained by applying a known deformation; middle: template matched to target. Top: T1/T1 registration; middle: $\mathrm{T} 1 / \mathrm{T} 2$; bottom: $\mathrm{T} 1 / \mathrm{PD}$.

object and target images), for 3 different ground truth deformations. All values are smaller than one voxel, with the most accurate results being obtained for T1/T1-matching. The overlap coefficients for WM, GM and CSF in the ground truth and recovered tissue maps are tabulated in table 2 The results are visualized in figure 2 and figure 3

\section{Discussion}

We present an algorithm for non-rigid multimodal image registration using a viscous fluid model by defining a force field that drives the deformation such that mutual information of corresponding voxel intensities is maximized. Our method is in fact the merger of the mutual information based registration functional presented in [5] with the viscous fluid regularization scheme of [3].

The joint intensity probability of the images to be matched is estimated using Parzen windowing and is differentiable with respect to the deformation field. The size of the Parzen windowing kernel needs to be properly chosen such that the criterion is a more or less smooth function of the deformation field. This choice is related to the image noise. For all experiments described above, the same kernel was used, indepedently of the multispectral nature of the images. In the current implementation, the extension of the Parzen estimator is automatically computed using a leave $\mathrm{k}$ out cross validation technique maximizing an empirical likelihood of the marginal densities [1011]. The impact of the Parzen windowing kernel on the registration process needs further investigation. 

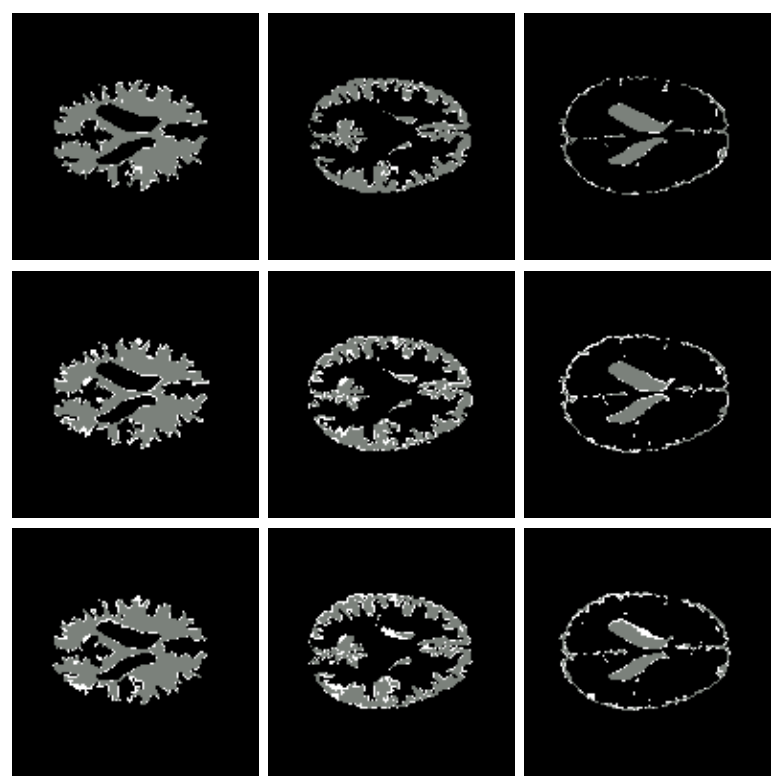

Fig. 3. Misclassified WM (left), GM (middle) and CSF (right) voxels of recovered vs ground truth deformation using the results in figure 2 . Top: T1/T1 registration; middle: T1/T2; bottom: T1/PD.

Another relevant implementation parameter is the time step $\Delta t$ or the maximal displacement $\Delta u$ allowed at each iteration that is specified to update the displacements after solving the Navier-Stokes equation. Selecting a larger value for $\Delta t$ will result in larger displacement steps and a more frequent regridding of the template as the Jacobian of the transformation is more likely to become non-positive. A smaller value of $\Delta t$ on the other hands implies a larger number of iterations for convergence. More experiments are needed to properly tune this parameter.

We validated our algorithm using simulated T1, T2 and PD images from BrainWeb with different noise levels and different realistic ground truth deformations generated by registration of the simulated image with real patient images. Although the RMS error was found to be subvoxel small in all cases, T1/T1 registration gave more accurate results than $\mathrm{T} 1 / \mathrm{T} 2$ or $\mathrm{T} 1 / \mathrm{PD}$ registration. The contrast between gray and white matter especially is much better in T1 than in $\mathrm{T} 2$ or $\mathrm{PD}$ and the algorithm succeeds better at recovering the interface between both tissues in T1 than in T2 or PD. We also compared T1-to-T2 versus T2-to$\mathrm{T} 1$ registration and found that somewhat better results are obtained using $\mathrm{T} 1$ as the template image. This can be explained by the fact that the forces driving the registration depend on the gradient of the template image, which is better defined in $\mathrm{T} 1$ than in $\mathrm{T} 2$ at the interface between white and gray matter. 


\section{Conclusions}

We have presented a multimodal free-from registration algorithm based on maximization of mutual information that models the images as a viscous fluid. The forces deforming the images are defined as the gradient of mutual information with respect to the deformation field, using Parzen windowing to estimate the joint intensity probability. We have validated our method for matching simulated T1-T1, T1-T2 and T1-PD images, showing that the method performs quite well in both mono and multi-modal conditions. Future work includes the introduction of more spatial information and more specific intensity models into the similarity criterion in order to make the registration more robust.

\section{References}

1. Available at http://www.bic.mni.mcgill.ca/brainweb/.

2. M. Bro-Nielsen, C. Gramkow. Fast Fluid Registration of Medical Images. Proc. Visualization in Biomedical Computing (VBC'96), Lecture Notes in Computer Science, vol. 1131, pp. 267-276, Springer, 1996.

3. G.E. Christensen, R.D. Rabitt, M.I. Miller. Deformable Templates Using Large Deformation Kinematics. IEEE Trans. Medical Imaging, 5(10):1435-1447, 1996.

4. T. Gaens, F. Maes, D. Vandermeulen, P. Suetens. Non-rigid multimodal image registration using mutual information. Proc. Medical Image Computing and Computer-Assisted Intervention (MICCAI98), Lecture Notes in Computer Science, vol. 1496, pp. 1099-1106, Springer, 1998.

5. G. Hermosillo, C. Chef d'Hotel, O. Faugeras. A Variational Approach to MultiModal Image Matching. INRIA Technical Report N. 4117, February 2001.

6. B. Likar, F. Pernus. A hierarchical approach to elastic registration based on mutual information. Image and Vision Computing, 19:33-44, 2000.

7. F. Maes, A. Collignon, D. Vandermeulen, G. Marchal, and P. Suetens. Multimodality image registration by maximization of mutual information. IEEE Trans. Medical Imaging, 16(4):187-198, 1997.

8. D. Rueckert, L.I. Sonoda, C. Hayes, D.L.G. Hill, M.O. Leach, D.J. Hawkes. Nonrigid registration using free-form deformation: application to breast MR images. IEEE Trans. Medical Imaging, 18(8):712-721, 1999.

9. K. Van Leemput, F. Maes, D. Vandermeulen, P. Suetens. Automated model-based tissue classification of MR images of the brain. IEEE Trans. Medical Imaging, 18(10):897-908, 1999.

10. G. Hermosillo Valadez. Variational methods for multimodal image matching. Doctoral Thesis, Universite de Nice, Sophia Antipolis, 138-141, 3 May 2002.

11. B. A. Turlach. Bandwidth selection in kernel density estimation: a review. Discussion Paper 9317, Institut de Statistique, UCL, Louvain La Neuve, 1993. 